\title{
A spectroscopic event of $\eta$ Car viewed from different directions: The data and first results ${ }^{\star}$
}

\author{
O. Stahl ${ }^{1}$, K. Weis ${ }^{2, \star \star}$, D. J. Bomans ${ }^{2}$, K. Davidson ${ }^{3}$, T. R. Gull ${ }^{4}$, and R. M. Humphreys ${ }^{3}$ \\ ${ }^{1}$ Landessternwarte Königstuhl, 69117 Heidelberg, Germany \\ e-mail: 0.Stahl@lsw.uni-heidelberg.de \\ 2 Astronomisches Institut, Ruhr-Universität Bochum, Universitätsstr. 150, 44780 Bochum, Germany \\ 3 Laboratory for Astronomy and Space Science, NASA-Goddard Space Flight Center, Code 681, Greenbelt, MD 20771, USA \\ 4 Astronomy Department, University of Minnesota, Minneapolis, MN 55455, USA
}

Received 15 December 2004 / Accepted 28 January 2005

\begin{abstract}
We present spectroscopic observations with high spectral resolution of $\eta$ Car as seen by the SE lobe of the Homunculus nebula over the 2003.5 "spectroscopic event". The observed spectra represent the stellar spectrum emitted near the pole of the star and are much less contaminated with nebular emission lines than direct observations of the central object. The "event" is qualitatively similar near the pole to what is observed in direct spectra of the star (more equator-on at $45^{\circ}$ ), but shows interesting differences. The observations show that the equivalent width changes of $\mathrm{H} \alpha$ emission and other lines are less pronounced at the pole than in the line of sight. Also the absorption components appear less variable. A pronounced high-velocity absorption is present near the event in the He I lines indicating a mass-ejection event. This feature is also seen, but less pronounced, in the hydrogen lines. He II $\lambda 4686$ emission is observed for a brief period of time near the event and appears, if corrected for light travel time, to precede similar emission in the direct view. Our observations indicate that the event is probably not only a change in ionization and excitation structure or a simple eclipse-like event.
\end{abstract}

Key words. stars: individual: $\eta$ Car - stars: circumstellar matter - stars: mass-loss - stars: evolution

\section{Introduction}

$\eta$ Car is one of the most massive and luminous stars of the Galaxy. During its "great eruption" observed around 1843 it was one of the brightest stars in the sky (e.g. Davidson \& Humphreys 1997). Today it is visually much fainter, largely because most of the UV and visual light is absorbed by dust from the surrounding nebula and re-radiated in the infrared (Westphal \& Neugebauer 1969; Cox et al. 1995). Its bolometric luminosity is still very high (Davidson 1999a). The surrounding nebula is visible as a bright reflection nebula, known as the Homunculus. Its expansion has been detected already in the 1950s (see Davidson \& Humphreys 1997, for references). Recent proper motion measurements indicate that most of this nebula was created during $\eta$ Car's "great eruption" (Currie et al. 1996; Smith \& Gehrz 1998; Morse et al. 2001).

The spectrum of $\eta$ Car is exceedingly complex. Seeinglimited ground-based spectra of the star are strongly contaminated by the very bright emission of the Weigelt blobs (Weigelt $\&$ Ebersberger 1986) and other ejecta close to $\eta$ Car. Only

* Based on observations obtained with UVES at the ESO Very Large Telescope, Paranal, Chile (proposals 70.D-0607(A), 71.D-0168(A), 072.D-0524(A)).

$\star \star$ Lise Meitner fellow.
STIS spectroscopy can separate the spectrum of the star from the nebular emission (Davidson et al. 1999; Gull et al. 1999).

The spectrum of $\eta$ Car is also known to be variable in time. Marked changes in the high-excitation lines of $\eta$ Car have been reported several times (see references cited by Damineli et al. 1999) before Damineli (1996) first noticed that these "spectroscopic events" recur in a 5.5-year cycle. The exact nature of this cycle is not yet understood. Most of the very strong variations seen in the emission lines of ground-based spectra of $\eta$ Car at the "spectroscopic event" (e.g. Zanella et al. 1984) are in fact not due to spectral variations of the central source but due to variations in the condensations close to the center (Hamann et al. 1999). A spectroscopic scan of the Homunculus was done by Hillier \& Allen (1992). They could show that most the emission lines in the outer Homunculus are scattered from the central region and only some are intrinsic to the Homunculus.

The cycle is also observed as eclipse-like event in the X-rays (Ishibashi et al. 1999). In the visual range (Fernandez Lajus et al. 2003) and the near-IR (Whitelock et al. 2004) a short dip in the light-curve is also observed. In the radio range, structural changes and strong flux changes of the nebula with the same period are observed (Duncan \& White 2003). Martin \& Koppelman (2004) observed the cycle photometrically with HST and therefore could separate variations of the 
star from variations of its environment. At present, the cause of the cyclic variations is not known. While the exact periodicity suggests a binary explanation, it is not obvious that such a scenario can explain all the observed variations (Davidson 1999b). The spectroscopic changes are clearly not due to a simple eclipse. Ground-based spectroscopic studies over a complete cycle have been done (Damineli et al. 1998; Wolf et al. 1999; Damineli et al. 2000), but give little information on the changes in the central source. While the HST/STIS observations by Smith et al. (2003) and more recent observations by the $\eta$ Car HST treasury team (Davidson \& et al. 2004; Humphreys et al. 2004) provide the necessary spatial resolution, the temporal sampling of these observations is not as dense as desirable.

Due to the geometry of the nebula, the reflection in the Homunculus allows us to view the spectrum of $\eta$ Car from different latitudes above the star's surface. This is of particular interest, since HST/STIS long slit spectroscopy in 1998-2002 by Smith et al. (2003) did show that the star is not spherical and its wind is bimodal. These results provided new insights with crucial implications for the event, the nature of $\eta$ Car, its mass loss history and bipolar ejecta. Smith et al. (2003) find that the wind from the polar regions is fast and dense while it is slower and less dense at lower latitudes. The direct view on the star corresponds to a stellar latitude of about $45^{\circ}$. The strong anisotropy of the stellar wind of $\eta$ Car is also indicated by direct interferometric observations (van Boekel et al. 2003) in the near infrared, where the stellar wind can be directly resolved. van Boekel et al. (2003) find that the stellar wind is elongated in the direction of the axis of the Homunculus, indicating an alignment of the axis of the Homunculus with the rotation axis of the star.

Here we report ground-based VLT/UVES observations of the star's reflected spectrum observed in the south-east lobe of the Homunculus nebula. While these observations cannot match the spatial resolution of the HST, they have a denser sampling, significantly higher spectral resolution and excellent $\mathrm{S} / \mathrm{N}$-ratio. A short description of the data set has been given by Weis et al. (2004).

The Homunculus appears to be a very clumpy, thin bipolar shell with hollow interior. Smith (2002) notes that the near infrared molecular hydrogen emission is consistent with the shell thickness being about ten percent of its distance, $D$, from $\eta$ Car. Spectra recorded at the center of the foreground SE lobe, known as "FOS4", appear to represent the stellar spectrum emitted near the pole of the star (Davidson et al. 1995; Zethson et al. 1999). They are much less contaminated by nebular emission lines than seeing-limited ground-based observations of the star directly. As has been shown by Rivinius et al. (2001), ground-based spectra of FOS4 can therefore be used to study the spectral changes of the star itself through the cycle.

The UVES project presented here is coordinated with STIS observations. Some first results of the STIS Davidson et al. (2005) and UVES Weis et al. (2005) observations have been reported already. The purpose of this paper is to present the data and to give an overview of the spectroscopic variations. Due to the large size and complexity of the data set and the strong spatial and temporal variations, more detailed aspects will be discussed in follow-up papers.

\section{The observations}

The data presented here are based on observations carried out in service observing mode between December 2002 and March 2004 with UVES, the Ultraviolet and Visual Echelle Spectrograph at the Nasmyth platform B of ESO's VLT UT2 (Kueyen) on Cerro Paranal, Chile. For all observations the standard settings DIC1, 346+580 and DIC2, 437+860 were used. The observed wavelength range extends from about $3200 \AA$ to $10200 \AA$ except for small gaps due to the space between the two CCDs of the detector mosaic at the red channel. Since the main aim was the observation of the reflected light of the Homunculus, the slit was offset from the star 2".6 south and $2^{\prime \prime} 8$ east of the star and the slit was aligned along the SE lobe of the Homunculus at a position angle of $160^{\circ}$.

The exact location of the slit is shown in the paper by Weis et al. (2005).

The temporal sampling of our observations was quite dense close to the event (about one spectrum every two weeks) and more sporadic further away from the event. Unfortunately, $\eta$ Car is very low in the sky between August and November which made it unobservable with UVES. Therefore, the immediate recovery from the 2003.5 event was not covered by our observations. STIS observations were accomplished during this gap and will be discussed elsewhere.

In order to observe both the extremely strong $\mathrm{H} \alpha$ emission and fainter lines with good signal-to-noise ratio, two exposures with different exposure times were obtained for the observations with the DIC1, 346+580 setting. Typical exposure times are $10 \mathrm{~s}$ for the short and $15 \mathrm{~min}$ for the long exposure. The slit width for the observations was 0.3 and 0 . $^{\prime} 4$ in the red and blue range, respectively, resulting in a spectral resolution of about 800000 . The pixel scales and slit lengths were, respectively, $0.246 /$ pixel and $7{ }^{\prime} .6$ in the blue and $0 . ' 182 /$ pixel and $11^{\prime \prime} .8$ in the red arm. The observational data were reduced and two-dimensional spectra were extracted using mostly standard ESO pipeline software for UVES. An exception was the order-merging procedure where the pipeline software did not produce satisfactory results, mainly because the very noisy edges of the echelle orders deteriorated the $\mathrm{S} / \mathrm{N}$ in the overlapping regions. Therefore, the order merging was carried out using software developed at the LSW Heidelberg (Stahl et al. 1999). All spectral frames were converted to the same (heliocentric) wavelength scale.

As a reference for the spectrum before the "spectroscopic event", we also reduced and analyzed UVES spectra obtained during the UVES commissioning (Dec. 21, 1999), which are released to the community and have also been used by Rivinius et al. (2001). They have been obtained with the same wavelength setting as our spectra, but with a different slit orientation. The position angle of the slit was $45^{\circ}$, i.e. about perpendicular to the position angle of the Homunculus and the slit was offset by 4" SE from the star. The exposure time of the spectrum used was $60 \mathrm{~s}$. A figure with the exact location of the slit is shown by Weis et al. (2005).

Basic data for the spectra used are summarized in Weis et al. (2005). The phase interval from 0.9 to 0.127 around the "spectroscopic event" is covered by our observations. 
Table 1. Heliocentric radial velocities of [Fe II] at various positions lines along the slit. The offset is measured relative to the center of the slit. Offsets relative to the center of the $\eta$ Car are also given. The errors are of the order of $5 \mathrm{~km} \mathrm{~s}^{-1}$. The velocities at the centers of the extraction positions are also given.

\begin{tabular}{lllll}
\hline \hline Offset ["] & Vel. $\left[\mathrm{km} \mathrm{s}^{-1}\right]$ & RA ["] & Dec ["] & Pos. index \\
\hline 1 & 80 & 2.95 & 3.75 & \\
1.5 & 92 & 3.10 & 4.20 & \\
2 & 112 & 3.30 & 4.70 & \\
2.5 & 141 & 3.45 & 5.15 & \\
3 & 178 & 3.65 & 5.60 & \\
3.5 & 224 & 3.80 & 6.10 & \\
4 & 279 & 4.00 & 6.60 & \\
4.5 & 341 & 4.15 & 7.00 & \\
\hline 0 & 80 & 2.60 & 2.80 & “1” \\
0.95 & 80 & 2.95 & 3.70 & “2” \\
1.90 & 107 & 3.25 & 4.60 & “3” \\
2.85 & 166 & 3.60 & 5.50 & “4” \\
3.80 & 255 & 3.90 & 6.40 & “5” \\
\hline
\end{tabular}

Unfortunately, due to observing constraints, there is a significant gap between phase 0.016 and 0.074 . Another smaller gap is present between phase 0.946 and 0.970 , but this is at a less critical phase.

In order to study the temporal variations at specific positions in the nebula, we have extracted one-dimensional spectra from the long-slit spectra at five positions in the Homunculus. The part of the slit which is close to the center shows a very complex spectrum and significant amount of scattered light (due to seeing) from the central source. For the present study, we therefore used only positions located at center of the slit (about 2.' 6 south and 2'. 8 east of the central star) and in steps of 0.95 outwards along the slit. We call these position "1" to " 5 " in the following. Position " 2 " is the brightest position and close to the center of the Homunculus, where already Humphreys \& The HST-FOS $\eta$ Car Team (1999) noticed that a very clear stellar spectrum can be observed. This position is also known as FOS4 in the literature (The FOS4 position was originally defined as a 0.5 region $4^{\prime \prime} .03$ from the center at a position angle of $135^{\circ}$. Correcting for the expansion of the Homunculus, it would now be at distance of 4'.21). Five or three rows of the CCD have been averaged in the red and blue spectral range, respectively, in order to increase the $\mathrm{S} / \mathrm{N}$-ratio of the extracted spectra. As reference for the spectrum close to the object, we also extracted one position close to the star, denoted as " 0 ". The extracted CCD rows lines have been corrected for the velocity shift (see below and Table 1) before averaging, with the exception of the spectra at position " 0 ", which have not been corrected.

For better visualization, we also present the data in the form of time series spectra, where the spectra, using the routines

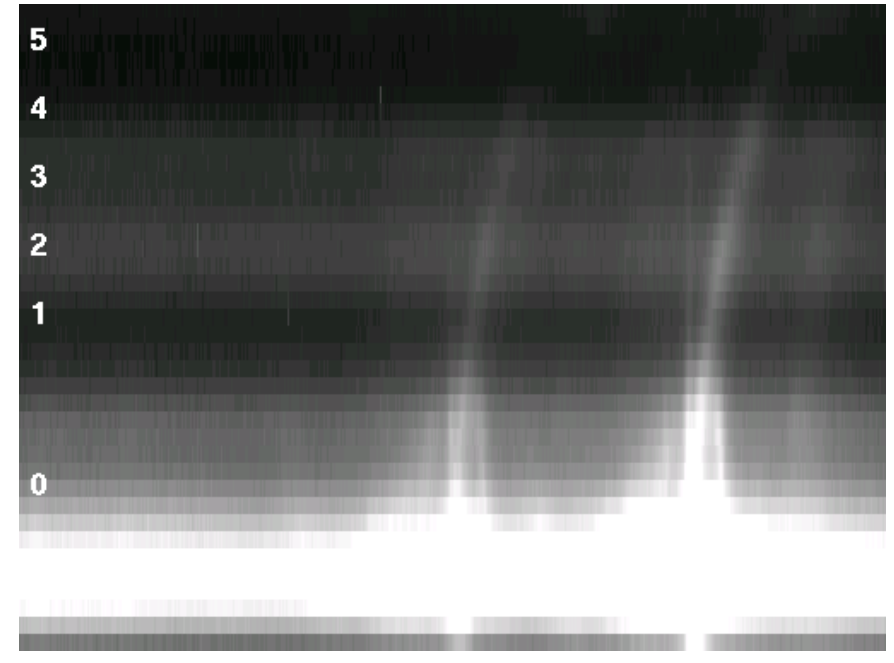

Fig. 1. Long-slit spectrum around the [Fe II $] \lambda \lambda 4277,4287$ lines. The image covers $37 \AA$ in wavelength and 9!' 3 along the slit. Note the complex velocity structure below the center of the slit. The approximate extraction positions are indicated. Here we discuss mainly the region above the center of the slit.

described by Kaufer et al. (1996), are interpolated along the time axis to form a "dynamical spectrum" When we extract information from the spectra, we do not use these interpolated spectra, but the extracted spectra directly.

\section{Basic data}

\subsection{Period and phase}

Slightly different values of the zero point and period of the cyclic variations are given in the literature. The differences are small and not relevant for the present paper. In the following we shall use the period and zero point of Steiner \& Damineli (2004), defined by the disappearance of He I $\lambda 6678$, of $P=2022.1$ days and $T_{0}=2452819.8$.

\subsection{Velocity shifts}

The reflection in the Homunculus causes a wavelength shift and a light-travel time delay. The spectral lines in the reflected spectra appear shifted in wavelength because of the expansion of the Homunculus (Thackeray 1961). These wavelength shifts have to be corrected in order to compare spectra extracted along the slit. We measured the velocity shifts in spectra taken well before the spectroscopic event in Dec. 2002, because they contain sharp forbidden emission lines, which appear most suitable for this measurement. We used several [Fe II] lines, which are relatively strong outside the event. As an example we show in Fig. 1 a small spectral range around the [Fe II] $\lambda 4287$ line.

The heliocentric radial velocity has been measured for several lines and averaged. The mean values have been fitted by a polynomial as a function of the distance from the slit center. The polynomial values for several positions along the slit are given in Table 1. The errors are of the order of $5 \mathrm{~km} \mathrm{~s}^{-1}$. For the velocity correction we used the polynomial values at 
the individual CCD rows to correct each row individually. This avoids the smearing due to different velocity shifts when averaging CCD rows.

In order to determine the velocity shift due to reflection, these velocity have still to be corrected for the velocity of the emitting gas in the direction from the star to the scattering surface. The systemic velocity of $\eta$ Car has been estimated to $-7 \pm 10 \mathrm{~km} \mathrm{~s}^{-1}$ by Davidson et al. (1997) by measuring the velocities of $\mathrm{O}$ stars near $\eta \mathrm{Car}$. This is in agreement with more recent measurement of Smith (2004), who used $\mathrm{H}_{2}$ measurements of the Homunculus nebula to obtain $-8.1 \pm 1 \mathrm{~km} \mathrm{~s}^{-1}$. The [Fe II] lines likely originate in compact emission regions close to the central star, the so-called "Weigelt blobs". These regions have a complex velocity structure (Smith 2004) and are moving in the equatorial plane of the nebula (Davidson et al. 1997). Their velocities towards the SE lobe, which are relevant here, are probably small. We therefore used the heliocentric velocity shifts without further correction. All velocities quoted below and all extracted one-dimensional spectra are corrected for this velocity shift.

\subsection{Time delay}

The light-travel time delay is caused by the difference in paths between direct line of sight and the longer path from the emitter to the scattering surface to the observer. This time delay can be related to the velocity shift. In the simplest model of the Homunculus nebula, the time delay depends linearly on the velocity shift. This model assumes that the expansion velocity of the Homunculus is unchanged since the eruption and the Homunculus is a hollow reflection nebula.

Meaburn et al. (1987) derived the following expression for the velocity shift caused by the reflection at the surface of the nebula:

$v_{\text {shift }}=(1-\cos \theta) v_{\text {dust }}$.

The time delay, on the other hand is given by:

$t_{\text {delay }}=(1-\cos \theta) D_{\text {Homunculus }} / c$.

Assuming a constant expansion velocity of the nebula, we have:

$D_{\text {Homunculus }}=v_{\text {dust }} \times t_{\text {Homunculus }}$

and the following relation between the velocity shift $v_{\text {shift }}$, the speed of light $c$ and the delay time $t_{\text {delay }}$ and the age of the Homunculus $t_{\text {Homunculus }}$ can be derived:

$v_{\text {shift }} / c=t_{\text {delay }} / t_{\text {Homunculus }}$.

This relation directly links the velocity shift and the time delays. For $t_{\text {delay }}$ in days and $v_{\text {shift }}$ in $\mathrm{km} \mathrm{s}^{-1}$, we get then for an expansion age of the Homunculus of $160 \mathrm{yr}$ :

$t_{\text {delay }}=0.195 \times v_{\text {shift }}$.

I.e., for a velocity shift of $100 \mathrm{~km} \mathrm{~s}^{-1}$ in the direction of FOS4, the light-travel delay time is about 20 days.

The exact time and temporal evolution of the "spectroscopic event" may depend on stellar latitude. Before we can draw any conclusions about this dependence, the observation times have to be corrected for this light-travel time delay.

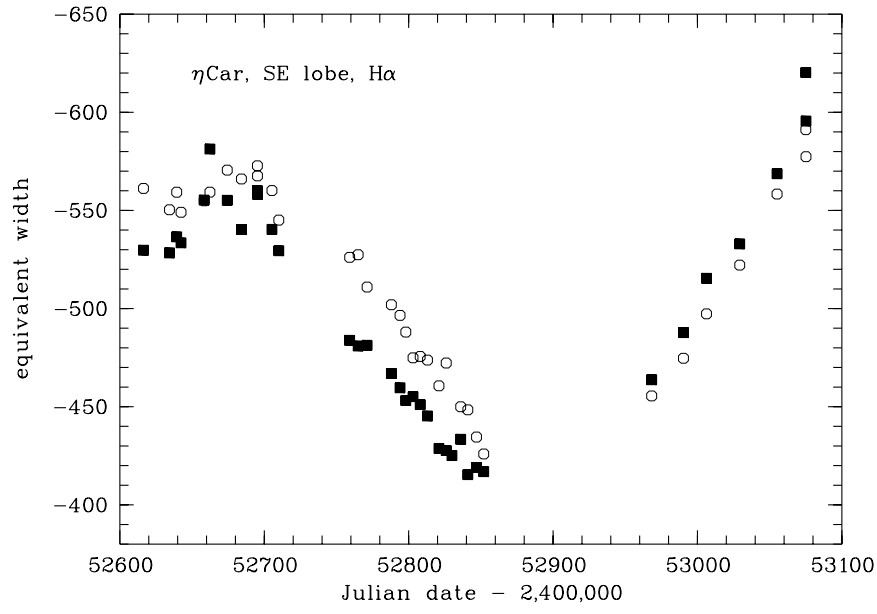

Fig. 2. Equivalent width versus Julian Date for position "2" (filled symbols) and position "5" (open symbols). The curve at position "5" seems delayed, but the time delay is smaller after the event.

\section{Variations in different lines}

\subsection{Hydrogen Balmer and Paschen lines}

The detailed behaviour of the Balmer lines as observed at one particular position in the Homunculus (FOS4) has already been discussed by Weis et al. (2005). Here we add some additional information.

Figure 2 shows the equivalent width of $\mathrm{H} \alpha$, the brightest observable feature, between 2002 and 2004. The equivalent width has been measured between 6500 and $6620 \AA$. The decrease of the equivalent width during the event is clearly visible. The decrease of equivalent width during the event appears later at position " 5 ", as expected because of the longer time delay. The increase after the event is less delayed, however, indicating that the delay is not only a light travel effect. In spite of the significant difference in line profiles, the equivalent width does not change strongly along the slit. The equivalent width is, however, always significantly smaller (by almost a factor of two) than observed directly on the star (Davidson et al. 2005). Also the amplitude of the variations is smaller than in STIS spectra taken on the central source (Martin \& Koppelman 2004). The decrease of the equivalent width stars around JD 2452700 (phase 0.94) and the recovery is still ongoing at the end of our observations at phase 0.127 .

Seen directly of $\eta$ Car, the Balmer line absorption strongly increases at the event (Davidson et al. 2005). By contrast, the Balmer line absorption in the polar spectra, as seen at FOS4 position, always has P Cygni absorption.

The line profiles of the Paschen lines, as an exemplified by Paschen 8 in Figs. 3 and 4 behave in a similar manner to the Balmer lines, but with a more pronounced red bump. The FOS Paschen 8 line profiles (Figs. 3 and 4) have an absorption strength that increases rapidly at the event with the edge velocity increasing significantly up to about $-800 \mathrm{~km} \mathrm{~s}^{-1}$. The high velocities appear at JD 2452800 (phase 0.990) and are strongest around JD 2452830 (phase 1.005). The increase of edge velocity is especially clear when comparing with spectra 


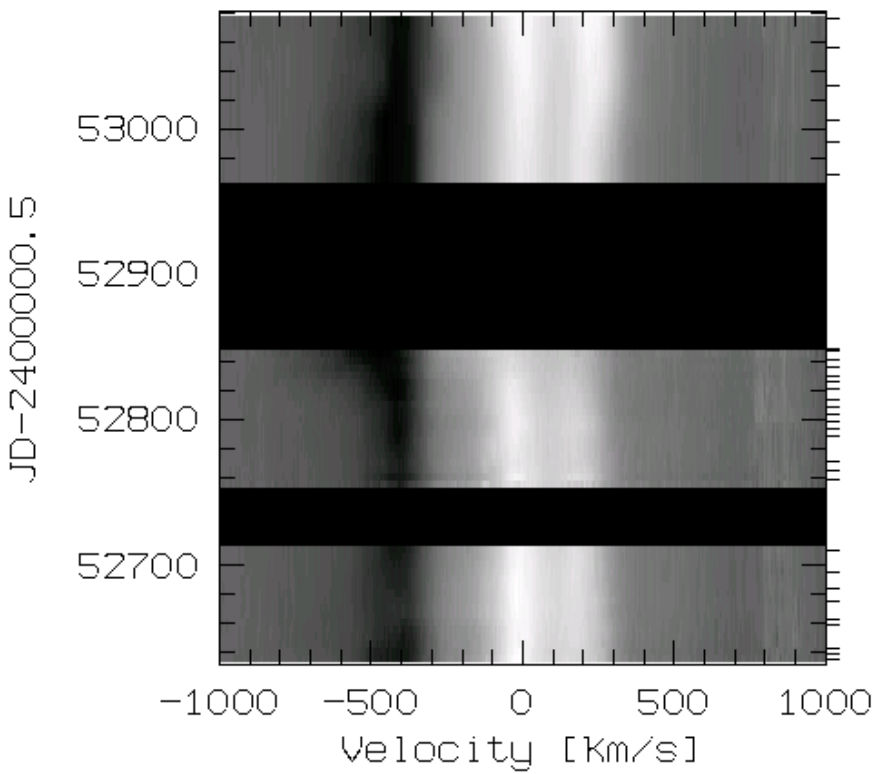

Fig. 3. Time series spectrum of Paschen 8 , as derived from the observed spectra. Dates are given in JD-2 400 000. The dates where spectra have been taken are marked at the right. Note the strong increase in edge velocity near JD 2452830 . The red bump is always very pronounced in the Paschen lines.

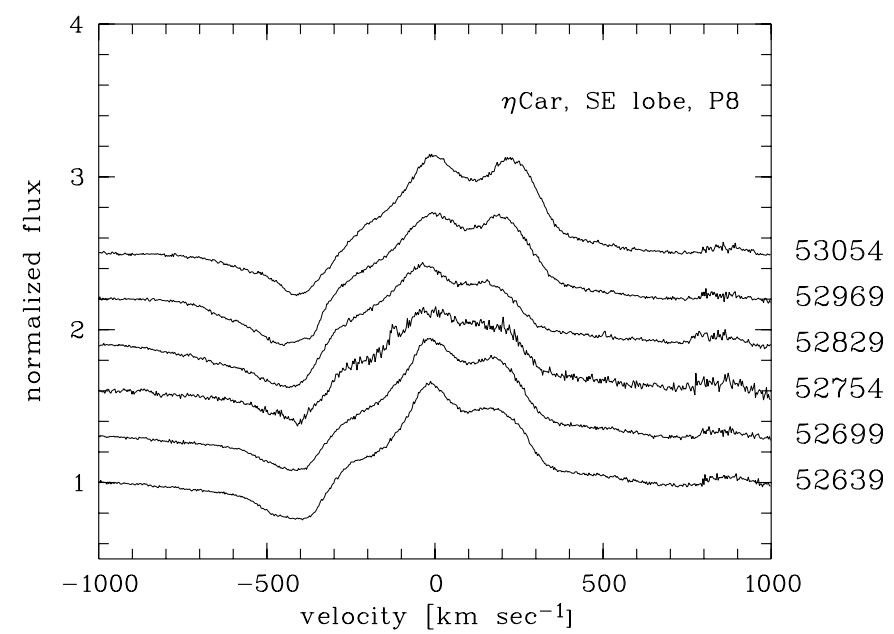

Fig. 4. Selected spectra from the data set used in Fig. 3. The spectra used have been taken close to the beginning and end of the observing run and close to the gaps in the observing runs. Note the strong increase in edge velocity close to the "event" and the strong red bump.

obtained after the event. Before the event, the absorption edge is very smooth and therefore ill-defined. At the phase of maximum edge velocity, the emission strength also seems to reach a minimum value, although this is not completely clear due to the gap shortly after after the event. The main peak has a velocity close to zero. After the event, a hump is visible in the red at a velocity of about $+200 \mathrm{~km} \mathrm{~s}^{-1}$. The velocity of the bump is not constant but varies between about +150 and $+220 \mathrm{~km} \mathrm{~s}^{-1}$. The edge velocity is also variable and reaches a maximum of at least $-800 \mathrm{~km} \mathrm{~s}^{-1}$.

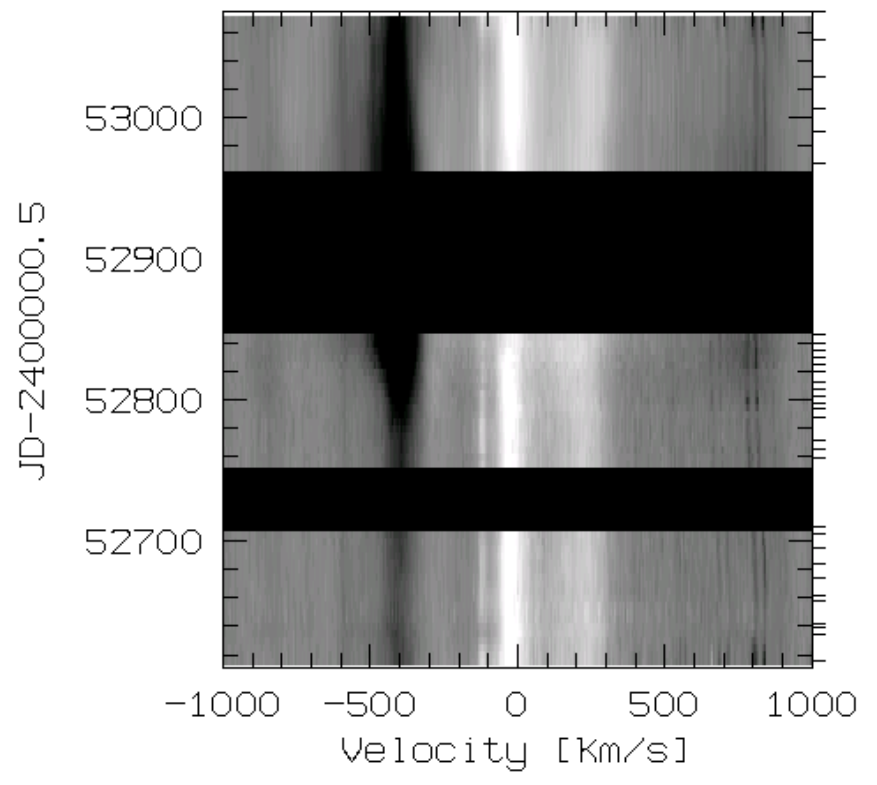

Fig. 5. Same as Fig. 3, but for Fe II $\lambda 6456$. The emission is relatively constant. The absorption increases strongly at the event and remains strong for an extended period. Recovery is not yet completed at the end of our observing run. High-velocity absorption similar to the hydrogen lines is not seen.

\subsection{Fe II lines}

Strong Fe II and [Fe II] lines are well-known features in the direct spectrum of $\eta$ Car. In the reflected light, the [Fe II] emission lines, narrow emission originating from nebular structures close to $\eta$ Car, are much fainter than seen in the direct spectrum of the star and close nebular structure. We discuss temporal variations seen by the FOS4 position (Figs. 5 and 6) of the Fe II $\lambda 6456$ line here as representative of low ionization metal lines. The absorption in this line increases strongly (starting around JD 2452800 - phase 0.990), but, in contrast to the hydrogen lines, without a significant increase of the edge velocity. The strong absorption persists for an extended period of time, long after the event, until the end of our monitoring. The profile of the Fe II line is qualitatively similar to the Paschen lines, with a pronounced red emission peak. The main peak is sharp and at a velocity of about zero, the red peak is broader and peaks at about $+220 \mathrm{~km} \mathrm{~s}^{-1}$.

The time evolution of Fe II $\lambda 6456$ changes strongly along the slit position in the Homunculus. In Fig. 7 we show the equivalent width variations at two different positions. While for both positions the absorption strength is stronger after the event, the effect is increases further out on the slit and thereby with angle from the polar axis of the SE lobe. In addition, the decrease begins at position "2" before the event (phase 0.97), but we do not see the decrease at position " 5 ". Only the fact that the equivalent width is less after the event indicates a sharper and later drop in equivalent width. 


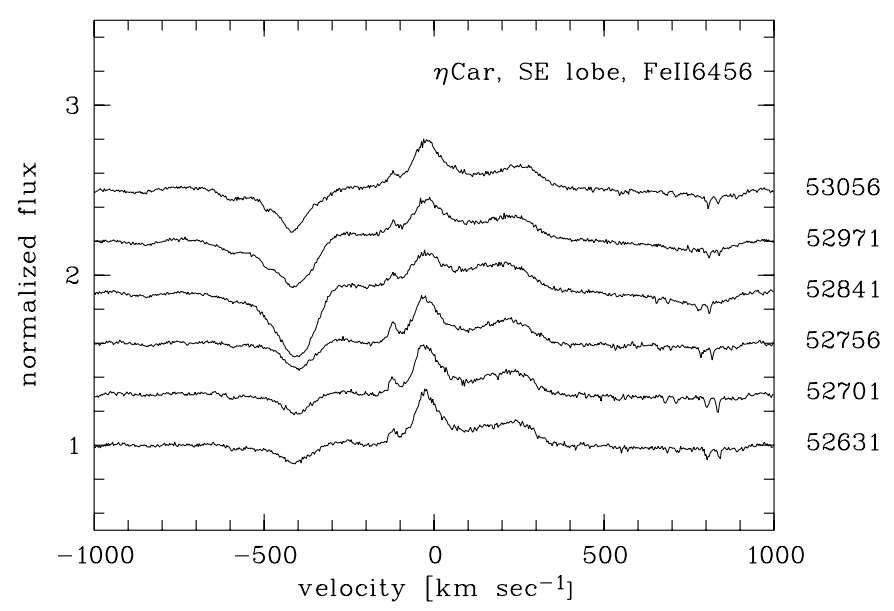

Fig. 6. Same as Fig. 4, but for Fe II $\lambda 6456$. The emission is relatively constant. The absorption increases strongly at the event and remains strong for an extended period. High-velocity absorption is not seen.

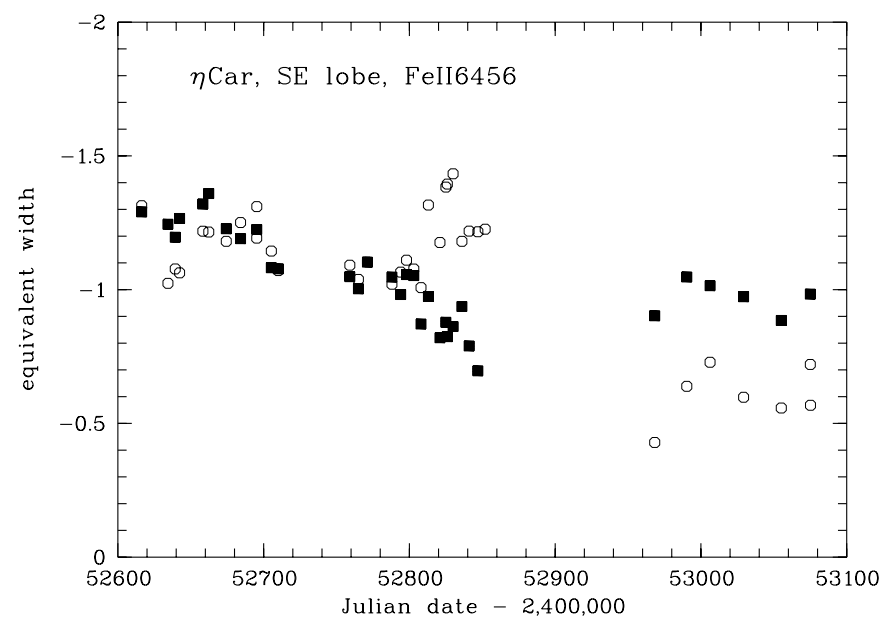

Fig. 7. Equivalent width of Fe II $\lambda 6456$ versus Julian Date for position "2" (filled symbols) and position " 5 " (open symbols). The equivalent width at " 5 " does not drop before the event. However, after the event, the absorption is stronger than at position " 2 ".

\subsection{He I lines}

The strong decrease in the strength of He I emission is characteristic for the event. Figures 8 and 9 show time variations in the He I 26678 line. It appears that a flux minimum occurs around JD 2452 844. In addition, a brief episode of high-velocity absorption (edge velocity about $-750 \mathrm{~km} \mathrm{~s}^{-1}$ at JD 2452830 , corresponding to phase 0.005) is seen in Fig. 8. The absorption strength decreases after the event.

Figures 10 and 11 show the same for the He I $\lambda 7065$ line. This line shows less absorption than the HeI 26678 line, but also this line shows evidence for a brief episode of highvelocity absorption.

The evolution of the equivalent width of He I $\lambda 7065$ is shown in Fig. 12. The change in equivalent width is dramatic. The equivalent widths outside of the event are similar at position " 2 " and " 5 ", but, as expected, the change is delayed further out on the slit. However, the difference cannot be attributed only to the time delay, since the curves are also intrinsically

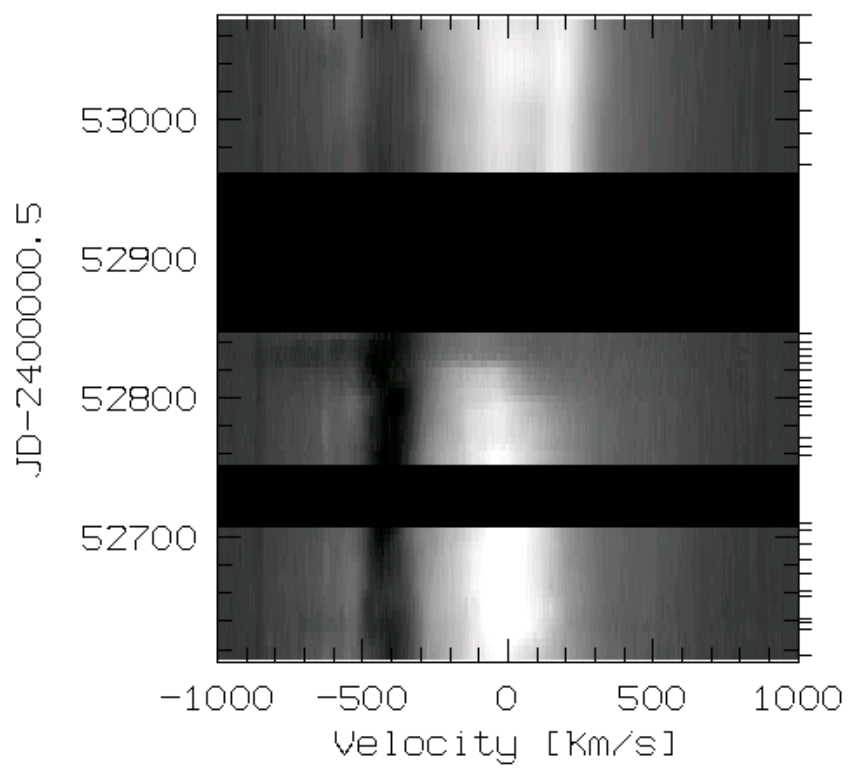

Fig. 8. Same as Fig. 3, but for He $\mathrm{I} \lambda 6678$. The emission is strongly variable and nearly disappears at the event. Note the brief episode of a high-velocity wind at the same time. A red-shifted emission bump develops after the event.

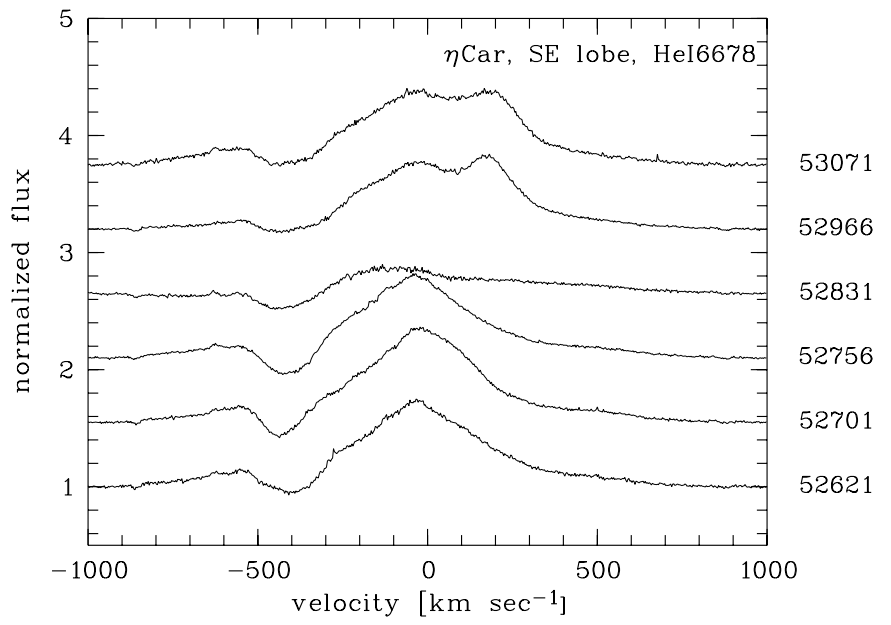

Fig. 9. Same as Fig. 4, but for He I $\lambda 6678$.

different: The residual equivalent width appears smaller at position "2" (corresponding to light emitted close to the pole), which indicates somewhat smaller changes there. This may be an artefact of the observing gap, however, since the minimum at position " 5 " is expected in this gap.

\subsection{He $11 \lambda 4686$}

He II $\lambda 4686$ is an important temperature diagnostic for hot stars. The absence of this line has been used by Hillier et al. (2001) to put constraints on the effective temperature of $\eta$ Car. However, Steiner \& Damineli (2004) detected He II $\lambda 4686$ in groundbased spectra of $\eta$ Car for a brief episode close to the event. Later, this line was also detected by Martin et al. (2004) in STIS spectra centered on the star. The He II $\lambda 4686$ emission 


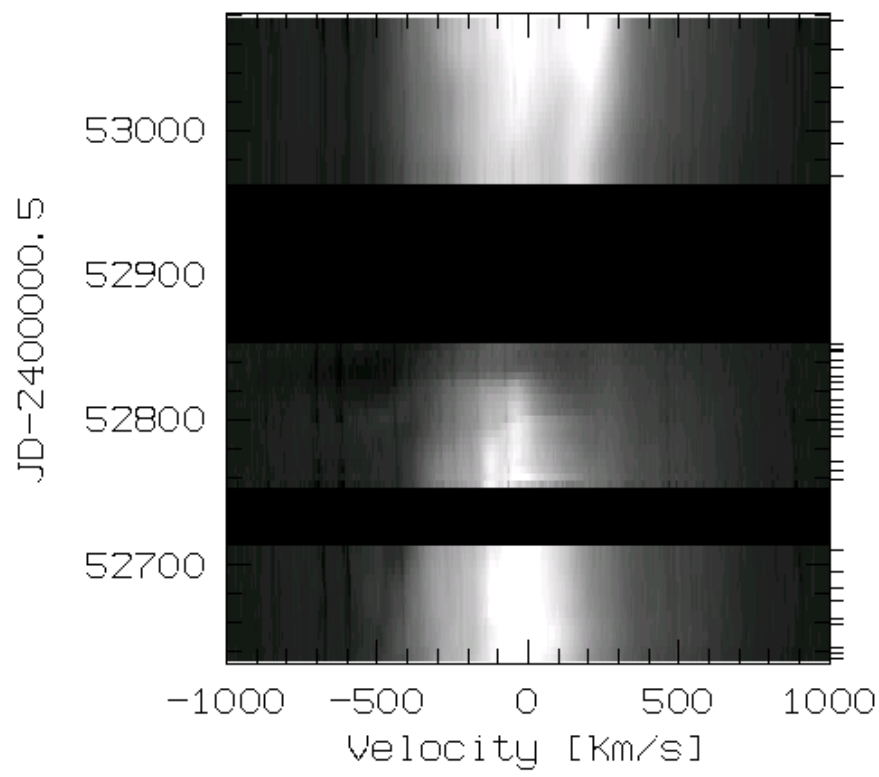

Fig. 10. Same as Fig. 3, but for He I $\lambda 7065$. The emission is strongly variable and nearly disappears at the event. In this line, absorption is always very weak, except for a brief episode of a high-velocity wind at the event. A red-shifted emission bump develops after the event.

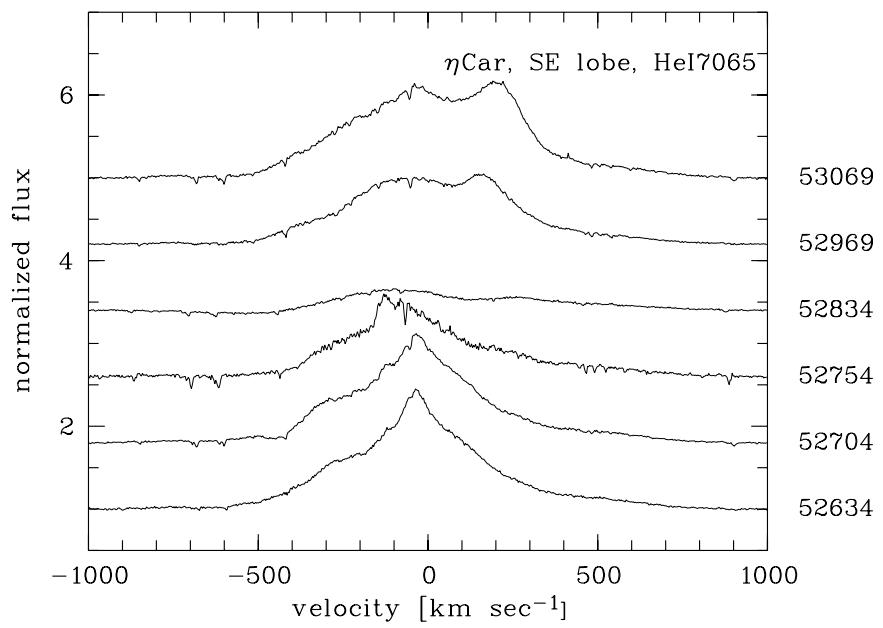

Fig. 11. Same as Fig. 4, but for He $\mathrm{I} \lambda 7065$.

seems to originate in a shock front. We find He II $\lambda 4686$ also in the reflected spectrum (cf. Figs. 13, 14).

The emission is highly variable and clearly visible only for a very brief period (about one month) around phase zero. It peaks around JD 2452815 (phase 0.998) and disappears again around JD 2452835 (phase 1.008). We do not detect this line clearly outside the event. Surprisingly, the peak of the flux shows a time delay of only about 10 days compared to the observations of Steiner \& Damineli (2004), which have been taken centered on the star. The observations of Martin et al. (2004) show the highest equivalent width almost exactly at the same date as our spectra. In their spectra, the equivalent width is, however, almost zero at 2452825 , while we still measure a significant equivalent width at 2452830 . Because of the special importance of this line, we also measured its equivalent

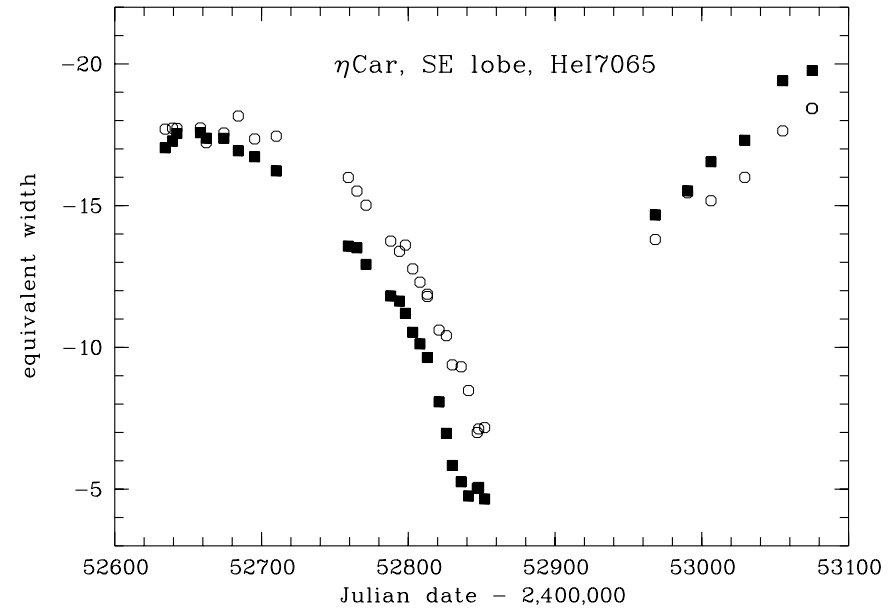

Fig. 12. Equivalent width of He I $\lambda 7065$ versus Julian Date for position " 2 " (filled symbols) and position " 5 " (open symbols). The curve at position " 5 " is delayed by about 30 days and the variations are somewhat smaller relative to position " 2 ".

width close to the star (see below). The measured values at position "2" and "0" are shown in Fig. 16. This plot shows that the rise and fall of the emission is reasonably well sampled with our spectra. The emission feature is broad and faint, therefore the $\mathrm{S} / \mathrm{N}$ in the equivalent width measurement is quite low. At larger distance the $\mathrm{S} / \mathrm{N}$ is still lower and the emission can barely be measured. We conclude that the peak of our measurements is somewhat delayed compared to direct spectra, but the delay appears to be limited to about ten days.

As observed in spectra of the center, the He II emission is significantly blue-shifted. The maximum equivalent width observed is about $500 \mathrm{~m} \AA$, about a factor of two lower than in ground-based spectra of the central source (Steiner \& Damineli 2004) and still smaller than in STIS spectra taken directly on the star (Martin et al. 2004).

At positions further out on the slit, the emission is also present, but appears, as expected, later in the cycle. It should be stressed, however, that the sampling of our spectra is insufficient to determine the exact date of the peak in He II emission better than within about two weeks.

Since He II $\lambda 4686$ has a high diagnostic value, we also extracted the spectrum closer to the central source. The dynamical spectrum extracted 2".5 inwards from the center of the slit is shown in Fig. 15. Here the blue-ward motion of the emission peak is clearer than in Fig. 13, possibly as a result of higher S/N. This shift has already been found and discussed by Steiner \& Damineli (2004). The tick marks at the right side in Fig. 15 show the dates where spectra have been taken. This shows that the peak is reasonably well sampled, but obviously some data are interpolated. The equivalent widths are shown in Fig. 16. The peak is stronger closer to the center and also peaks earlier by about 10 days.

\section{Discussion and conclusion}

Our UVES observations show in significant detail the evolution of line profiles during a spectroscopic event in reflected light. 


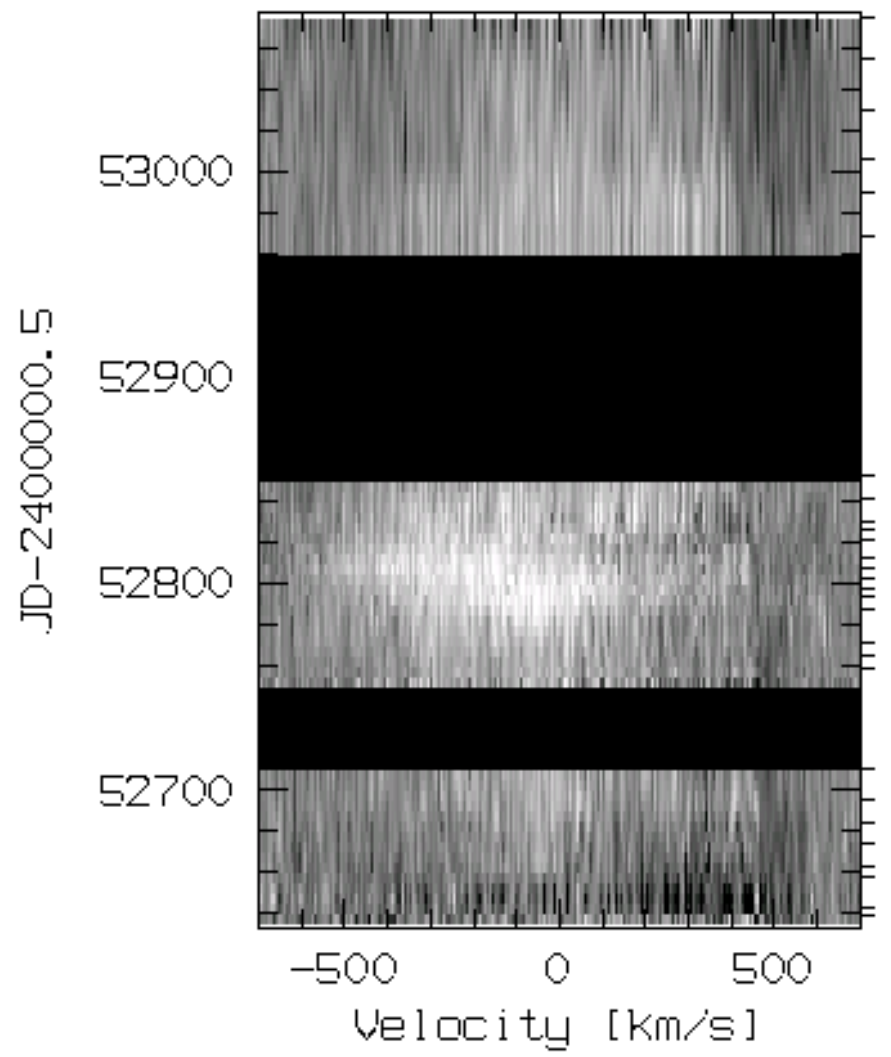

Fig. 13. Same as Fig. 3, but for He II $\lambda 4686$. Faint emission appears to be present well before the event, but briefly increases strongly close to the event. The emission disappears again just after phase zero. The emission is blue-shifted and appears to move to the blue with time.

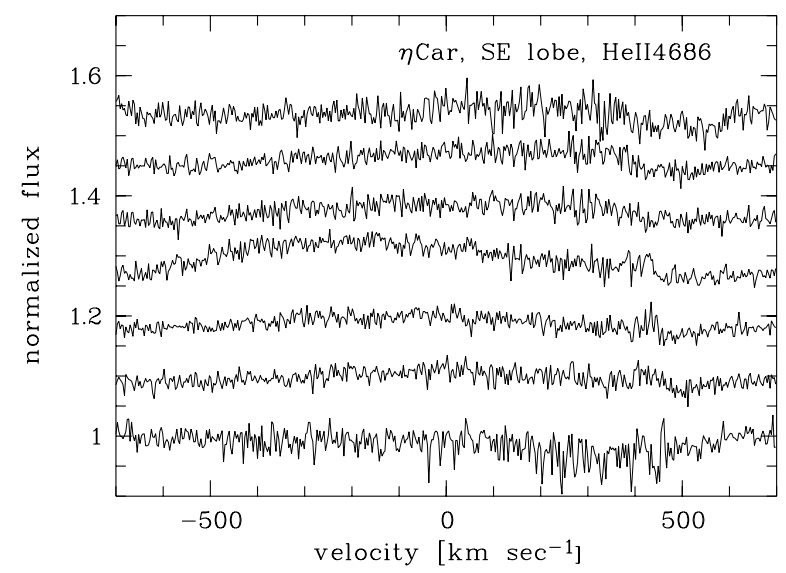

53069

52964

52849

52809

52764

52704

52644

Fig. 14. Same as Fig. 4, but for He II $\lambda 4686$.

Assuming that the emission observed at FOS4 is only a scattered spectrum originating from the central source and does not include emission from other sources, these observations correspond to an about pole-on view of $\eta$ Car, while the direct observations of the star see it at about $45^{\circ}$. While many changes are qualitatively similar to what was already known from observations of the central source, there are some important differences. We have also significantly better temporal sampling, spectral resolution and S/N than Smith et al. (2003).

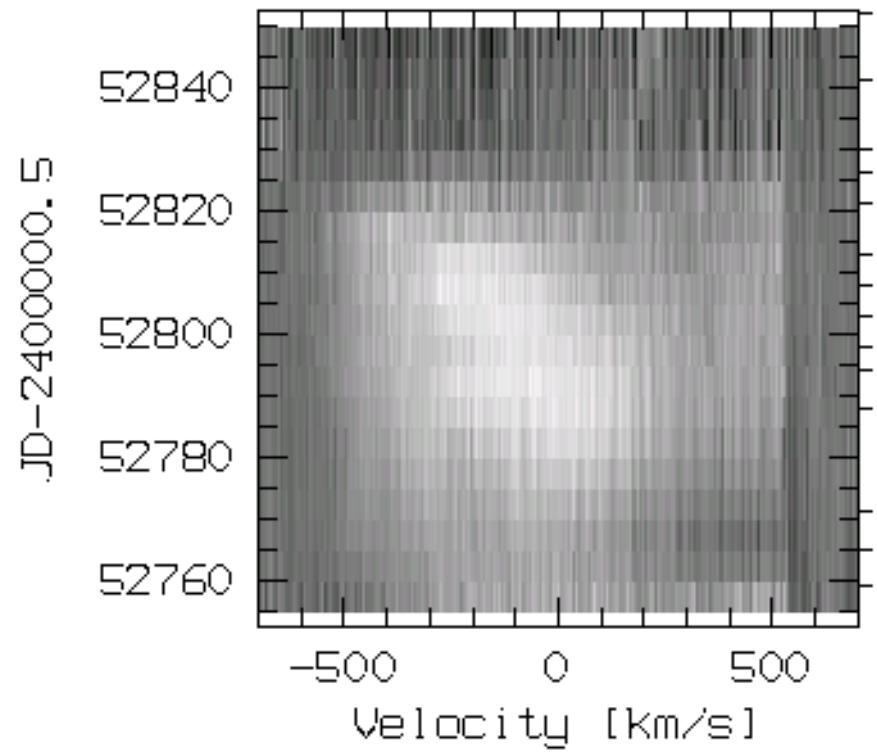

Fig. 15. Same as Fig. 3, but for He II $\lambda 4686$ extracted close to the center of the Homunculus. The emission is blue-shifted and appears to move to the blue with time. The apparent absorption at the right side is a artefact due to the order merging. This is a close-up covering only the well-sampled part close to the event. The tick marks at the right side show the dates where spectra have been taken.

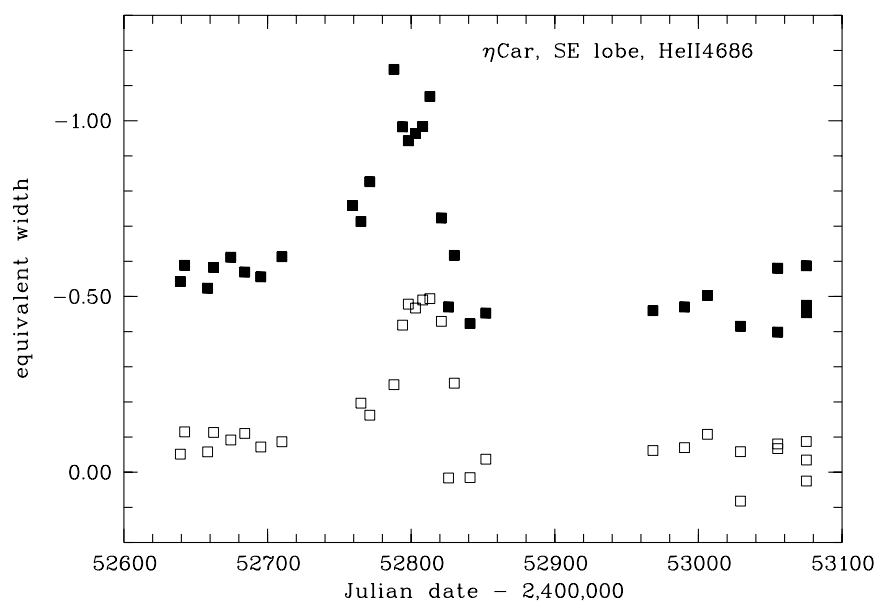

Fig. 16. Equivalent width of He II $\lambda 4686$ versus time as measured at position " 2 " (open symbols) and closer to the center (position "0", filled symbols). The latter are shifted up to 0.5 units for clarity. The peak emission is stronger close to the center.

The Balmer lines have already been discussed in detail by Davidson et al. (2005) and Weis et al. (2005). The decrease of the equivalent width of the hydrogen lines during the spectroscopic event is pronounced, but not as strong as seen in the direct line of sight (Davidson et al. 2005). The equivalent width is always significantly smaller than observed directly on the star.

The appearance of a strong red bump in the reflected spectra has already been described for the Balmer lines by Weis et al. (2005). We see the same feature in many different lines, in particular after the event. This feature is not a peculiarity of the present observations, but also present in spectra obtained in Dec. 1999 during UVES commissioning (Weis et al. 2005). 
At present, the origin of this component and its relation with the spectroscopic cycle is not clear.

The changes in the high-excitation lines such as He I are particularly pronounced. The equivalent widths change dramatically and, in addition, a high-velocity absorption appears very briefly near the event. High-velocity absorption has also been observed in $\mathrm{H} \alpha$ (Weis et al. 2005), but since this absorption appears long after the spectroscopic event, it is not clear if it is related to the event.

The low-ionization metal lines such as Fe II show, similar to the hydrogen lines, an increasing absorption during the event, but no evidence for high-velocity absorption. For these lines, the recovery phase after the event seems particularly extended. These lines also seem to have a complicated dependence on the viewing angle.

He II $\lambda 4686$ line emission is seen in the spectrum of the SE lobe, consistent with viewing the polar region, but with significantly smaller equivalent width than seen of the star directly. If corrected for light-travel time delay, the polar emission of the He II $\lambda 4686$ appears to peak earlier than closer to the equator $\left(45^{\circ}\right)$, constraining models for the origin of this emission.

The He II $\lambda 4686$ line is only one example of how the geometry of the region where the event occurs can be constrained by comparing the timing of the event as seen in reflected light in the Homunculus and in direct spectra. In practice this is complicated by several factors:

- The observations of the reflected spectra have to be corrected for the light-travel time delay.

- With the UVES data, only the beginning of the event is well sampled. The recovery phase is sampled incompletely because of observing constraints.

- The STIS data are also not very densely sampled.

- The qualitative temporal behaviour of e.g. the equivalent widths is significantly different between positions in the SE lobe of the Homunculus and direct spectra. This makes it difficult to derive a simple timing difference.

Only detailed quantitative modeling of the event can at least partly resolve these problems. However, already the equivalent width variations measured in UVES spectra of the Homunculus at different slit positions clearly indicate that the event depends on the viewing angle.

The spectral changes of the hydrogen lines and even more the He I and Fe II lines are clearly not symmetric in time. The "ingress" phase is more rapid than the "egress" phase. The changes can be described as a "shell-like" event, i.e. a fast onset and a slow recovery phase. Such a shell-like event is especially supported by a new feature not described before: A phase of high-velocity absorption, which appears very briefly near the event especially in the He I lines. This is one of the strongest indications that the spectroscopic event involves some mass ejection and is not simply due an eclipse event.

A more detailed comparison of the reflected spectra taken at different slit positions and with the STIS spectra obtained directly at the center will be the subject of following papers.
Acknowledgements. It is a pleasure to thank the ESO Paranal Observatory staff for carrying out for us the service mode observations on which this paper is based.

\section{References}

Cox, P., Mezger, P. G., Sievers, A., et al. 1995, A\&A, 297, 168

Currie, D. G., Dowling, D. M., Shaya, E. J., et al. 1996, AJ, 112, 1115

Damineli, A. 1996, ApJ, 460, L49

Damineli, A., Kaufer, A., Wolf, B., et al. 2000, ApJ, 528, L101

Damineli, A., Stahl, O., Kaufer, A., et al. 1998, A\&AS, 133, 299

Damineli, A., Stahl, O., Wolf, B., Kaufer, A., \& Jablonski, F. J. 1999, in Eta Carinae at The Millennium, ed. J. A. Morse, R. M. Humphreys, \& A. Damineli, ASP Conf. Ser., 179, 221

Davidson, K. 1999a, in Eta Carinae at The Millennium, ed. J. A. Morse, R. M. Humphreys, \& A. Damineli, ASP Conf. Ser., 179, 6

Davidson, K. 1999b, in Eta Carinae at The Millennium, ed. J. A. Morse, R. M. Humphreys, \& A. Damineli, ASP Conf. Ser., 179, 304

Davidson, K., \& Humphreys, R. M. 1997, ARA\&A, 35, 1

Davidson, K., Ebbets, D., Weigelt, G., et al. 1995, AJ, 109, 1784

Davidson, K., Ebbets, D., Johansson, S., Morse, J. A., \& Hamann, F. W. 1997, AJ, 113, 335

Davidson, K., Ishibashi, K., Gull, T. R., \& Humphreys, R. M. 1999, in Eta Carinae at The Millennium, ed. J. A. Morse, R. M. Humphreys, \& A. Damineli, ASP Conf. Ser., 179, 227

Davidson, K., et al. 2004, in The Fate of the Most Massive Stars, ed. R. M. Humphreys, \& K. Stanek, ASP Conf. Ser., in press

Davidson, K., Martin, J., Humphreys, R. M., Ishibashi, K., et al. 2005, $\mathrm{AJ}$, in press

Duncan, R. A., \& White, S. M. 2003, MNRAS, 338, 425

Fernandez Lajus, E., Gamen, R., Schwartz, M., et al. 2003, Inform. Bull. Var. Stars, 5477, 1

Gull, T. R., Ishibashi, K., Davidson, K., \& The Cycle 7 STIS Go Team 1999, in Eta Carinae at The Millennium, ed. J. A. Morse, R. M. Humphreys, \& A. Damineli, ASP Conf. Ser., 179, 144

Hamann, F., Davidson, K., Ishibashi, K., \& Gull, T. R. 1999, in Eta Carinae at The Millennium, ed. J. A. Morse, R. M. Humphreys, \& A. Damineli, ASP Conf. Ser., 179, 116

Hillier, D. J., \& Allen, D. A. 1992, A\&A, 262, 153

Hillier, D. J., Davidson, K., Ishibashi, K., \& Gull, T. 2001, ApJ, 553, 837

Humphreys, R., Davidson, K., Gray, M., et al. 2004, in The Fate of the Most Massive Stars, ed. R. M. Humphreys, \& K. Stanek, ASP Conf. Ser.

Humphreys, R. M., \& The HST-FOS $\eta$ Car Team 1999, in Eta Carinae at The Millennium, ed. J. A. Morse, R. M. Humphreys, \& A. Damineli, ASP Conf. Ser., 107

Ishibashi, K., Corcoran, M. F., Davidson, K., et al. 1999, ApJ, 524, 983

Kaufer, A., Stahl, O., Wolf, B., et al. 1996, A\&A, 305, 887

Martin, J., Davidson, K., Ishibashi, K., \& et al. 2004, AJ, submitted

Martin, J. C., \& Koppelman, M. D. 2004, AJ, 127, 2352

Meaburn, J., Wolstencroft, R. D., \& Walsh, J. R. 1987, A\&A, 181, 333

Morse, J. A., Kellogg, J. R., Bally, J., et al. 2001, ApJ, 548, L207

Rivinius, T., Wolf, B., Stahl, O., \& Kaufer, A. 2001, in Eta Carinae and Other Mysterious Stars: The Hidden Opportunities of Emission Spectroscopy, ed. T. R. Gull, S. Johannson, \& K. Davidson, ASP Conf. Ser., 29, 29

Smith, N. 2002, MNRAS, 337, 1252

Smith, N. 2004, MNRAS, 351, L15

Smith, N., \& Gehrz, R. D. 1998, AJ, 116, 823 
Smith, N., Davidson, K., Gull, T. R., Ishibashi, K., \& Hillier, D. J. Weis, B., Bomans, D., Stahl, O., et al. 2004, in The Fate of the Most 2003, ApJ, 586, 432

Stahl, O., Kaufer, A., \& Tubbesing, S. 1999, in Optical and Infrared Spectroscopy of Circumstellar Matter, ed. E. W. Guenther, B. Stecklum, \& S. Klose, ASP Conf. Ser., 188, 331

Steiner, J. E., \& Damineli, A. 2004, ApJ, 612, L133

Thackeray, A. D. 1961, The Observatory, 81, 99

van Boekel, R., Kervella, P., Schöller, M., et al. 2003, A\&A, 410, L37

Weigelt, G., \& Ebersberger, J. 1986, A\&A, 163, L5 Massive Stars, ed. R. M. Humphreys, \& K. Stanek, ASP Conf. Ser. Weis, B., Stahl, O., Bomans, D., et al. 2005, AJ, in press

Westphal, J. A., \& Neugebauer, G. 1969, ApJ, 156, L45

Whitelock, P. A., Feast, M. W., Marang, F., \& Breedt, E. 2004, MNRAS, 352, 447

Wolf, B., Kaufer, A., Stahl, O., \& Damineli, A. 1999, in Eta Carinae at The Millennium, ASP Conf. Ser., 179, 243

Zanella, R., Wolf, B., \& Stahl, O. 1984, A\&A, 137, 79

Zethson, T., Johansson, S., Davidson, K., et al. 1999, A\&A, 344, 211 